\title{
Irinotecan-based combination chemotherapy for metastatic small intestinal adenocarcinoma
}

\author{
YOSHIHIRO SHIBATA, EISHI BABA, HIROSHI ARIYAMA, SHUJI ARITA, TAICHI ISOBE, \\ HITOSHI KUSABA, KENJI MITSUGI, SHUJI NAKANO and KOICHI AKASHI
}

First Department of Internal Medicine and Department of Biosystemic Science of Medicine, Kyushu University Graduate School of Medical Sciences, Fukuoka, Japan

Received December 9, 2009; Accepted February 26, 2010

DOI: 10.3892/ol_00000074

\begin{abstract}
Metastatic small intestinal adenocarcinoma (SIA) is rare among digestive tract malignancies, and a standard therapy has yet to be established. The present study described a patient who was treated with irinotecan-based chemotherapy. A 67-year-old woman with a long history of anemia was diagnosed as having SIA using small bowel endoscopy. Tumor invasion of the mesentery and multiple metastases to the lungs and peritoneal lymph nodes were detected. Nine courses of chemotherapy, each consisting of bolus infusion of 5-fluorouracil at $500 \mathrm{mg} / \mathrm{m}^{2}$, plus infusion of irinotecan at $100 \mathrm{mg} / \mathrm{m}^{2}$ with 1-leucovorin at $20 \mathrm{mg} / \mathrm{m}^{2}$ on days 1,8 and 15 , were administered at 4-weekly intervals postoperatively. After two courses, the metastatic nodules in the lungs showed a decrease in number and size, and this response continued for over 6 months. Adverse events were manageable during this period. The patient succumbed to the disease 12 months after the initial diagnosis. The present results therefore suggest that irinotecan-based chemotherapy is a potential treatment for metastatic SIA.
\end{abstract}

\section{Introduction}

Malignant tumors of the small intestine account for only $2.1 \%$ of all malignant tumors of the gastrointestinal tract in Japanese patients and are seldom encountered in clinical practice (1-3). While adenocarcinoma and carcinoid tumors comprise $80 \%$ of the primary malignant tumors of the small intestine in the US, adenocarcinoma and malignant lymphoma constitute 47 and 30\%, respectively, in Japan (4).

In most patients with small intestinal adenocarcinoma (SIA), the disease is already advanced by the time of diagnosis.

Correspondence to: Dr Eishi Baba, First Department of Internal Medicine and Department of Biosystemic Science of Medicine, Kyushu University Graduate School of Medical Sciences, 3-1-1 Maidashi, Higashi-ku, Fukuoka 812-8582, Japan

E-mail: e-baba@intmed1.med.kyushu-u.ac.jp

Key words: small intestinal adenocarcinoma, chemotherapy, irinotecan, 5-fluorouracil
Delay of the diagnosis is due to non-specific presentation, lack of awareness of the disease and inaccessibility of the tumor to clinical examination. The clinical staging reflects this delay, with stages I to IV being reported to account for 4, 20, 39 and $35 \%$, respectively. The 5-year survival rate of patients with stage I-III disease has been reported to be $36 \%$, compared to $5 \%$ for stage IV disease, and the median survival time of stage IV patients is only 11 months (5).

While surgical resection of the primary tumor and the regional lymph nodes is the preferred treatment for patients in stages I-III, chemotherapy is administered to patients with stage IV disease. However, a standard chemotherapy regimen for SIA has yet to be established, and the regimens employed are usually those designed for advanced colorectal or gastric adenocarcinoma (6-10). The clinical effectiveness of such regimens for the two latter cancer types is well established; nevertheless, their efficacy for the treatment of SIA has yet to be determined.

This study described a patient who was diagnosed with SIA with lung and lymph node metastases plus invasion of the transverse colon. She was treated with surgery and irinotecanbased chemotherapy, resulting in a partial response of the disease.

\section{Case report}

A 67-year-old woman consulted her doctor in 2002 complaining of fatigue and headache. As her blood count showed hypochromic anemia (hemoglobin $4.4 \mathrm{~g} / \mathrm{dl}$ ) and her stool was positive for occult blood, bleeding from the digestive tract was suspected. However, gastroduodenal endoscopy and colonoscopy revealed no abnormalities. Since her anemia persisted, these endoscopic examinations were repeated in August 2003 along with chest computed tomography (CT), abdominal CT and a gynecologic examination. A uterine myoma was diagnosed, and she was given a blood transfusion and an oral iron supplement. In August 2004, repeat endoscopy again revealed no abnormalities. The patient had lost $12 \mathrm{~kg}$ of weight over the preceding 4 years, and began to complain of abdominal pain, after which she was referred to our department. The study was prepared following ethics guidelines of clinical study issued by the Ministry of Health, Labor and Welfare of Japan. 
A

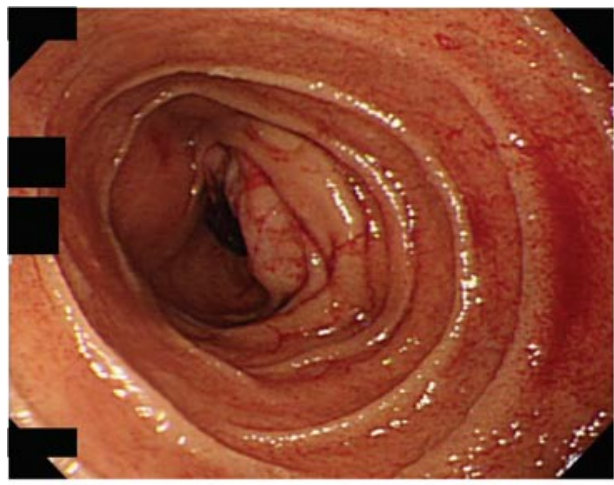

C

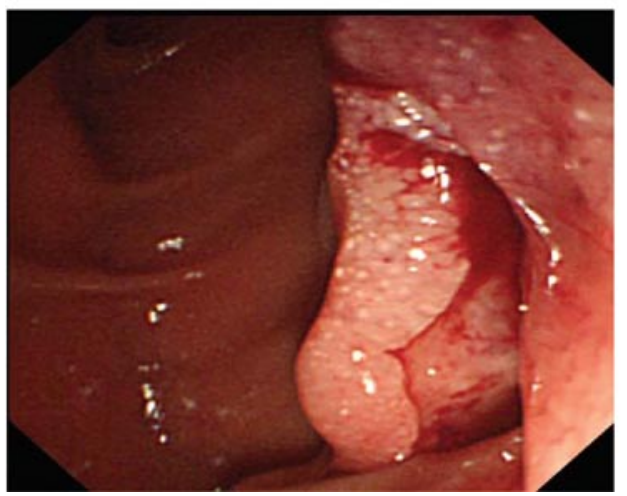

B

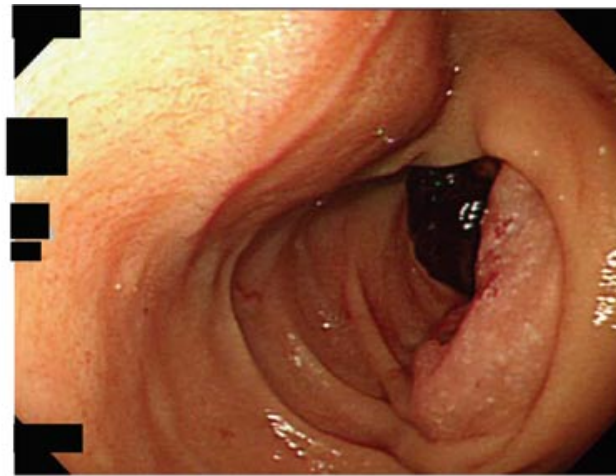

D

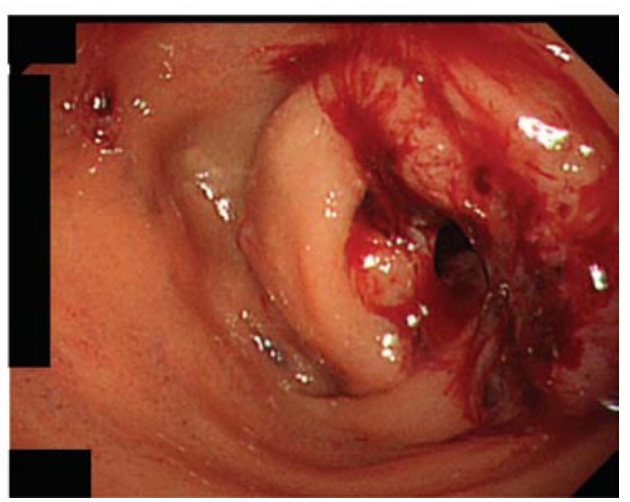

Figure 1. (A and B) Findings of the initial upper gastrointestinal endoscopy. (C and D) Second examination using side- and oblique-view endoscopy.

A
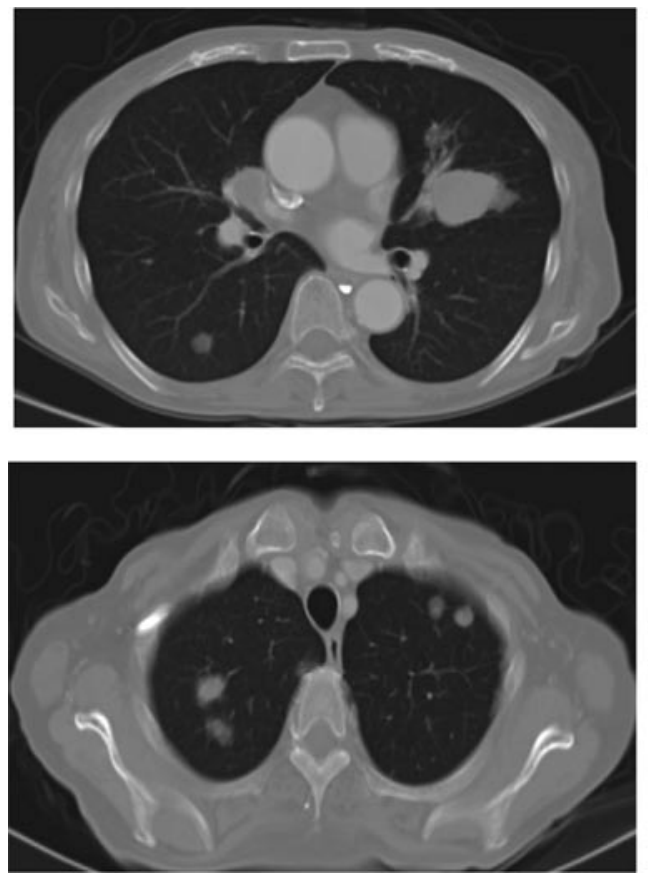

B
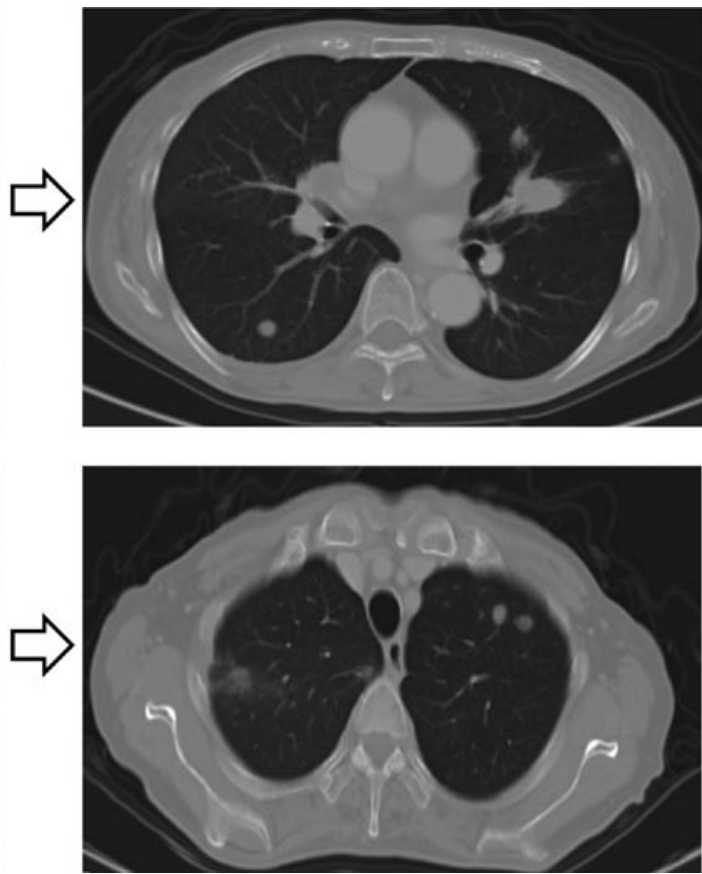

Figure 2. Serial chest computed tomography (CT) showing (A) lung metastases on admission and (B) tumor regression (65\%) after two courses of chemotherapy.

Upper gastrointestinal endoscopy revealed a large ulcerated tumor covered with clots in the distal third of the duodenum (Fig. 1A and B). A clear border was observed between the tumor and the surrounding mucosa. Side and oblique endoscopic views showed that the tumor occupied almost the entire lumen and was causing obstruction (Fig. 1C and D). Histological examination of a biopsy specimen showed that the tumor was a well- to moderately differentiated adenocarcinoma. 
A barium meal showed that the distal part of the tumor had an overhanging edge. Abdominal CT scans revealed a mass near the ligament of Treitz and invasion of the mesentery. The patient's chest X-ray film and CT scans showed multiple metastatic nodules in the lungs, but no lymphadenopathy. A blood count and bone marrow biopsy showed iron-deficiency anemia, and her plasma C-reactive protein level was $4.87 \mathrm{mg}$ / dl. The plasma levels of tumor markers (carcinoembryonic antigen and CA 19-9) were within normal limits. The results of other biochemical analyses showed no abnormalities.

Radical resection of this invasive tumor was impossible; thus, partial removal of the involved upper jejunum and duodenojejunostomy was performed to decrease the risk of obstruction. No peritoneal dissemination or liver metastases were noted. The postoperative course was uneventful. One month after the operation, chemotherapy was commenced, consisting of infusion of irinotecan (CPT-11) at $100 \mathrm{mg} / \mathrm{m}^{2}$ and l-leucovorin (l-LV) at $20 \mathrm{mg} / \mathrm{m}^{2}$, plus bolus infusion of 5-fluorouracil (5-FU) at $500 \mathrm{mg} / \mathrm{m}^{2}$ on days 1,8 and 15 . Administration on day 15 was postponed as grade 2 leukopenia was detected on day 14. After one course of chemotherapy, no non-hematological toxicities were noted, and a chest X-ray film showed that the lung metastases had shrunk. Due to persistent leukopenia, after the second course of chemotherapy, the dose of CPT-11 was reduced to $80 \mathrm{mg} / \mathrm{m}^{2}$ on days 1 and 8 every 3 weeks. Grade 2 leukopenia recurred during the subsequent courses, but was quickly resolved. Two courses of chemotherapy produced $65 \%$ tumor regression (Fig. 2), which was maintained over six courses. However, regrowth was observed after the six courses, leading to obstruction of the jejunum. The patient succumbed to the disease in December 2004, 12 months after the initial diagnosis.

\section{Discussion}

Chemotherapy is administered to treat stage IV SIA when metastases or the location of the tumor renders the tumor inoperable. The median survival time of stage IV patients is only 11 months.

Recently, Overman et al reported a retrospective analysis of 80 patients with primary SIA (11). They found that the response rate and progression-free survival were superior in patients treated with 5-FU plus platinum compared to those administered with 5-FU alone (46 vs. $16 \%$, and 8.7 vs. 3.9 months, respectively). Another retrospective study of 44 SIA patients treated with various chemotherapy regimens showed a median response rate of $29 \%$ and survival time of 18.6 months (12).

Few prospective studies exist for the treatment of SIA. A phase II study of 5-FU, doxorubicin and mitomycin C (FAM) showed that the response rate was $18 \%$ and the median survival time was 8 months (13). Combination chemotherapy with capecitabine and oxaliplatin in a phase II trial achieved a response rate of $50 \%$ and median survival time of 20.3 months (11). These reports suggest that combination chemotherapy that employs new anticancer agents may improve the outcome of patients with SIA.

Most of the chemotherapy regimens for SIA investigated thus far have been based on those designed for colorectal or gastric adenocarcinoma, while irinotecan-based chemo- therapy has not been widely used. In the above-mentioned retrospective study of Overman et al, only 2 patients received irinotecan (14). Ono et al reported that only 1 out of 8 SIA patients treated with irinotecan plus cisplatin achieved partial response (15). Two small phase I studies have also been reported. These studies investigated 5-FU, cisplatin and irinotecan in 5 patients as well as 5-FU, oxaliplatin and irinotecan in 4 patients, respectively. Thus, the efficacy of irinotecanbased regimens for SIA remains to be established. It is likely that both irinotecan- and platinum-based chemotherapy are equally effective against SIA.

When we initiated chemotherapy for the present patient in 2004, the standard first-line regimen for metastatic colorectal cancer (CRC) was a bolus 5-FU and irinotecan based on survival benefit (16), albeit that this regimen has been found to cause serious gastrointestinal toxicity. We previously conducted phase I (17) and phase II (18) clinical studies in a patient with metastatic CRC to establish the feasibility and effectiveness of employing a modified irinotecan and bolus 5-FU regimen. In these studies, CPT-11 $\left(100 \mathrm{mg} / \mathrm{m}^{2}\right)$ and bolus 5-FU $\left(500 \mathrm{mg} / \mathrm{m}^{2}\right)$ plus $1-\mathrm{LV}\left(20 \mathrm{mg} / \mathrm{m}^{2}\right)$ were administered on days 1,8 and 15 every 28 days, and a response rate of $53.3 \%$ for patients without prior chemotherapy was achieved (18). As a result, we found that a modified irinotecan-based regimen was promising for metastatic $\mathrm{CRC}$, and we selected this modified regimen for the treatment of the patient in the present study. Six courses of this therapy were administered, and the patient survived for 12 months. Her clinical response was very similar to that noted previously, and the low level of adverse events allowed the regimen to be administered safely $(5,7,8,10,19,20)$.

The previous standard irinotecan-based regimen for metastatic CRC, i.e., irinotecan and bolus 5-FU, has now been replaced by FOLFIRI (5-FU, irinotecan and leucovorin) due to its improved efficacy and safety profile $(21,22)$. Therefore, a modified irinotecan and bolus 5-FU regimen may also be an option for patients who are not able to receive continuous infusion of 5-FU. The results of the present study suggest that an irinotecan-based regimen is effective against SIA. A prospective phase II study of irinotecan combined with other appropriate agents may lead to a more effective treatment for metastatic SIA.

\section{References}

1. Sager GF: Primary malignant tumors of the small intestine. A twenty-two year experience with thirty patients. Am J Surg 135: 601-603, 1978.

2. Ciccarelli O, Welch JP and Kent GG: Primary malignant tumors of the small bowel. The Hartford Hospital experience, 1969-1983. Am J Surg 153: 350-354, 1987.

3. Jemal A, Murray T, Ward E, et al: Cancer statistics. CA Cancer J Clin 55: 259, 2005.

4. Kusumoto H, Takahashi I, Yoshida M, et al: Primary malignant tumors of the small intestine: analysis of 40 Japanese patients. J Surg Oncol 50: 139-143, 1992.

5. Dabaja BS, Suki D, Pro B, Bonnen $M$ and Ajani J: Adenocarcinoma of the small bowel: presentation, prognostic factors and outcome of 217 patients. Cancer 101: 518-526, 2004.

6. Polyzos A, Kouraklis G, Giannopoulos A, Bramis J, Delladetsima JK and Sfikakis PP: Irinotecan as salvage chemotherapy for advanced small bowel adenocarcinoma: a series of three patients. J Chemother 15: 503-506, 2003. 
7. Jigyasu D, Bedikian AY and Stroehlein JR: Chemotherapy for primary adenocarcinoma of the small bowel. Cancer 53: 23-25, 1984.

8. Onodera H, Nishitai R, Shimizu K, Maetani S and Imamura M: Small intestinal cancer with extensive lymph node metastases showing complete remission by methotrexate/5-fluorouracil sequential therapy: report of a case. Surg Today 27: 60-63, 1997.

9. Lioe TF and Biggart JD: Primary adenocarcinoma of the jejunum and ileum: clinicopathological review of 25 cases. J Clin Patho 43: 533-536, 1990.

10. Talamonti MS, Goetz LH, Rao S and Joehl RJ: Primary cancers of the small bowel: analysis of prognostic factors and results of surgical management. Arch Surg 137: 564-571, 2002.

11. Overman MJ, Kopetz S, Wen S, et al: Chemotherapy with 5-fluorouracil and a platinum compound improves outcomes in metastatic small bowel adenocarcinoma. Cancer 113: 2038-2045, 2008.

12. Fishman PN, Pond GR, Moore MJ, et al: Natural history and chemotherapy effectiveness for advanced adenocarcinoma of the small bowel: a retrospective review of 113 cases. Am J Clin Oncol 29: 225-231, 2006.

13. Gibson MK, Holcroft CA, Kvols LK and Haller D: Phase II study of 5-fluorouracil, doxorubicin and mitomycin $\mathrm{C}$ for metastatic small bowel adenocarcinoma. Oncologist 10: 132-137, 2005.

14. Overman MJ, Varadhachary GR, Kopetz S, et al: Phase II study of capecitabine and oxaliplatin for advanced adenocarcinoma of the small bowel and ampulla of Vater. J Clin Oncol 27 : 2598-2603, 2009.

15. Ono M, Shirao K, Takashima A, et al: Combination chemotherapy with cisplatin and irinotecan in patients with adenocarcinoma of the small intestine. Gastric Cancer 11: 251-255, 2008.
16. Saltz LB, Douillard JY, Pirotta N, Alakl M, Gruia G, Awad L, Elfring GL, Locker PK and Miller LL: Irinotecan plus fluorouracil/leucovorin for metastatic colorectal cancer: a new survival standard. Oncologist 6: 81-91, 2001.

17. Fujishima H, Kikuchi I, Miyanaga O, Ueda A, Baba E, Mitsugi K, Harada M and Nakano S: Phase I study of CPT-11 and bolus 5-FU/l-leucovorin in patients with metastatic colorectal cancer. Int J Clin Oncol 9: 92-97, 2004.

18. Fujishima H, Makiyama A, Miyanaga O, Ueda A, Esaki T, Mitsugi K, Baba E, Kusaba H, Harada $M$ and Nakano S: A multicenter phaseIIstudy of irinotecan (CPT-11) and bolus 5-fluorouracil (5FU)/1-leucovorin(l-LV) in patients with metastatic colorectal cancer. J Clin Oncol, 2005 ASCO Annual Meeting Proceedings. Vol. 23, No. 16, Part I of II, p3742, 2005.

19. Di Marco L, Berghenti M and Felloni M: Primary adenocarcinoma of the second portion of duodenum. Ann Ital Chir 74: 573-577, 2003.

20. Zhou Z, Wan D and Shi M: Diagnosis and treatment of primary malignant tumors of the small bowel. Zhonghua Zhong Liu Za Zhi 19: 297-299, 1997.

21. Goldberg RM, Sargent DJ, Morton RF, Fuchs CS Ramanathan RK, Williamson SK, Findlay BP, Pitot HC and Alberts SR: A randomized controlled trial of fluorouracil plus leucovorin, irinotecan and oxaliplatin combinations in patients with previously untreated metastatic colorectal cancer. J Clin Oncol 22: 23-30, 2004.

22. Tournigand C, Andre T, Achille E, Lledo G, Flesh M, Mery-Mignard D, Quinaux E, Couteau C, Buyse M, Ganem G, Landi B, Colin P, Louvet C and de Gramont A: FOLFIRI followed by FOLFOX6 or the reverse sequence in advanced colorectal cancer: a randomized GERCOR study. J Clin Oncol 22: 229-237, 2004. 